\title{
A relaxation scheme for the simulation of low Mach number flows
}

\author{
Emanuela Abbate, Angelo Iollo and Gabriella Puppo
}

\begin{abstract}
A scheme for the simulation of inviscid flows with low Mach number is derived. The scheme is built on a relaxation system and it is based on a linear implicit time discretization. The advective part is discretized by a convex combination of upwind and centered schemes, in order to recover the correct limit when the Mach number goes to zero. The implicit treatment allows to stabilize the central approximation in the low Mach limit and also to avoid demanding constraints on the time step in low Mach flows. The scheme applies to steady or unsteady flows and to general equations of state. We discuss examples pertaining to both gas and liquid flows.
\end{abstract}

Key words: low Mach flows, relaxation method, compressible flows

MSC (2010): 65M08, 65N08, 35Q31

\section{Introduction}

In the numerical simulation of fluid flows, the low Mach regime induces severe stiffness and, consequently, stability problems for standard computational techniques. This is due to the fact that the upwind schemes adopted when solving compress-

\footnotetext{
E. Abbate

Memphis team, Inria Bordeaux Sud-Ouest,

Univ. Bordeaux, IMB, UMR 5251, 33400 Talence, France

A. Iollo

Memphis team, Inria Bordeaux Sud-Ouest,

Univ. Bordeaux, IMB, UMR 5251,

33400 Talence, France

e-mail: angelo.iollo@u-bordeaux.fr

G. Puppo

Dip. di Scienza e Alta Tecnologia, Univ. dell'Insubria

via Valleggio, Como, Italy

e-mail: gabriella.puppo@uninsubria.it
}

Dip. di Scienza e Alta Tecnologia, Univ. dell'Insubria, via Valleggio, Como, Italy,

e-mail: eabbate.studenti@uninsubria.it,emanuela.abbate@u-bordeaux.fr,emanuela.abbate@ inria.fr 
ible flows are not "asymptotic preserving", namely they do not preserve the low Mach number limit behaviour, which tends to incompressible flow [6, 7]. It has been proved in [4] that upwind schemes lead to pressure fluctuations of the order of the Mach number $M$, while in the continuous case the pressure fluctuations are of order $M^{2}$ : this is one of the main reasons why compressible flow solvers perform so poorly in the low Mach number regime.

In this work, the derivation of a scheme for solving low Mach inviscid flows is addressed with a novel implicit relaxation approach. The relaxation method introduced in [5] is adopted, approximating the original system with a larger one. This new system is linear except for a lower order source term and thanks to linearity, implicit time discretizations can be easily implemented. Therefore, both upwind and centered spatial discretizations can be used without having stability problems. The centered scheme allows to recover the correct limit on the pressure gradients in the low Mach regime. Nevertheless, the upwind discretization is needed in presence of Mach numbers of order one, in order to introduce enough numerical viscosity. Since the adopted spatial discretization is a convex combination of these two, it is able to approximate flows in different regimes and thus the scheme can be seen as an "all-speed" scheme, as the ones proposed in $[2,3]$. Moreover, thanks to the absolute stability of implicit schemes, the CFL is not limited by the acoustic constraint, which becomes extremely demanding in low Mach regimes.

\section{The relaxation method}

We briefly revise the relaxation method developed in [5]. A general hyperbolic system of balance laws has the following structure:

$$
\partial_{t} \psi+\partial_{x} \mathbf{F}(\psi)=\mathbf{Q}(\psi),
$$

where $\psi \in \mathbb{R}^{n}$ is the vector of the conservative variables, $\mathbf{F}(\psi)$ the vector of the fluxes and $\mathbf{Q}(\psi)$ a source term. By introducing the relaxation variables vector $\mathbf{v} \in$ $\mathbb{R}^{n}$, the relaxation system approximating the original system (1) reads:

$$
\left\{\begin{array}{l}
\partial_{t} \psi+\partial_{x} \mathbf{v}=\mathbf{Q}(\psi) \\
\partial_{t} \mathbf{v}+\mathbf{A} \partial_{x} \psi=\frac{1}{\varepsilon}(\mathbf{F}(\psi)-\mathbf{v}), \quad \varepsilon>0,
\end{array}\right.
$$

where $\mathbf{A}=\operatorname{diag}\left\{a_{i}\right\}, i=1, \ldots, n$ is a positive diagonal matrix. The small positive parameter $\varepsilon$ is called relaxation rate. The right hand side of the second equation is a stiff lower order term. Our motivation in choosing this specific relaxation method relies in the resulting linearity of the advective part. This allows for an easy implementation of implicit schemes: the introduction of modified Riemann solvers is not required, as it is when adopting the Suliciu relaxation approach [1].

In order to ensure the dissipative nature of the relaxation, it is necessary to respect the Liu subcharacteristic condition (for details see [5]) 


$$
\mathbf{A}-\mathbf{F}^{\prime}(\psi)^{2} \geq 0 \quad \forall \psi
$$

when building the relaxation system. By enforcing this condition, the coefficients of the relaxation matrix $\mathbf{A}$ are derived in such a way that the characteristic speeds $\lambda_{i}, i=1, . . n$ of the original system (1) interlace with those of the relaxation system $\mu_{j}= \pm \sqrt{a_{i}}, j=1, . .2 n, i=1, . . n$. In our computations, $\mathbf{A}$ is built by taking $a_{i}=$ $\max _{x} \lambda_{i}^{2}$. This is the maximum over the domain of the a-priori estimated speed.

\section{Numerical relaxation scheme}

System (2) is discretized with finite volumes on a uniform mesh. For 1D problems, the grid spacing is $\Delta x=x_{i+1 / 2}-x_{i-1 / 2}$ and the time step $\Delta t=t_{n+1}-t_{n}$. Let $\mathbf{w}_{i}^{n}$ be the approximate cell average of a quantity $\mathbf{w}$ in the cell $\left[x_{i-1 / 2}, x_{i+1 / 2}\right]$ at time $t_{n}$ and $\mathbf{w}_{i+1 / 2}^{n}$ the approximate point value of $\mathbf{w}$ at an interface $x=x_{i+1 / 2}$ and at $t=t_{n}$.

In general, the spatial discretization for system (2) can be written as

$$
\left\{\begin{array}{l}
\partial_{t} \psi_{i}+\frac{\mathbf{v}_{i+1 / 2}-\mathbf{v}_{i-1 / 2}}{\Delta x}=\mathbf{Q}\left(\psi_{i}\right) \\
\partial_{t} \mathbf{v}_{i}+\mathbf{A} \frac{\psi_{i+1 / 2}-\psi_{i-1 / 2}}{\Delta x}=\frac{1}{\varepsilon}\left(\mathbf{F}\left(\psi_{i}\right)-\mathbf{v}_{i}\right)
\end{array}\right.
$$

This is valid with an accuracy of $\mathscr{O}\left(\Delta x^{2}\right)$ and for sufficiently accurate reconstructions of the quantities at the interfaces, noting that

$$
\mathbf{F}\left(\psi_{i}\right)=\mathbf{F}\left(\frac{1}{\Delta x} \int_{x_{i-1 / 2}}^{x_{i+1 / 2}} \psi d x\right)=\frac{1}{\Delta x} \int_{x_{i-1 / 2}}^{x_{i+1 / 2}} \mathbf{F}(\psi) d x+\mathscr{O}\left(\Delta x^{2}\right)=\mathbf{F}_{i}+\mathscr{O}\left(\Delta x^{2}\right) .
$$

\subsection{Spatial discretization}

In (4), the interface values have to be computed with a specific scheme. Let $\mathbf{u}$ be a generic variable. We define an hybrid discretization in the following way:

$$
\left(\frac{\mathbf{u}_{i+1 / 2}-\mathbf{u}_{i-1 / 2}}{\Delta x}\right)_{h y b}=f\left(M_{l o c}\right)\left(\frac{\mathbf{u}_{i+1 / 2}-\mathbf{u}_{i-1 / 2}}{\Delta x}\right)_{u p w}+\left(1-f\left(M_{l o c}\right)\right)\left(\frac{\mathbf{u}_{i+1 / 2}-\mathbf{u}_{i-1 / 2}}{\Delta x}\right)_{c e n t},
$$

where the first term defines the spatial discretization through an upwind method and the second through a centered one. $M_{l o c}$ is the local Mach number and we choose $f\left(M_{l o c}\right)=\min \left\{1, M_{l o c}\right\}$ in order to have a convex combination. This way the correct numerical viscosity for each regime is recovered.

The upwind scheme is derived as in [5], computing the interface values by using the characteristic variables $\mathbf{v} \pm \mathbf{A}^{1 / 2} \psi$ of system (2). At first order we have: 


$$
\left\{\begin{array}{l}
\left(\frac{\mathbf{v}_{i+1 / 2}-\mathbf{v}_{i-1 / 2}}{\Delta x}\right)_{u p w}=\frac{1}{2 \Delta x}\left(\mathbf{v}_{i+1}-\mathbf{v}_{i-1}\right)-\frac{\mathbf{A}^{1 / 2}}{2 \Delta x}\left(\psi_{i+1}-2 \psi_{i}+\psi_{i-1}\right) \\
\left(\frac{\psi_{i+1 / 2}-\psi_{i-1 / 2}}{\Delta x}\right)_{u p w}=\frac{1}{2 \Delta x}\left(\psi_{i+1}-\psi_{i-1}\right)-\frac{\mathbf{A}^{-1 / 2}}{2 \Delta x}\left(\mathbf{v}_{i+1}-2 \mathbf{v}_{j}+\mathbf{v}_{i-1}\right) .
\end{array}\right.
$$

For second order approximations, a Van Leer MUSCL scheme is employed. Since in this work we test second order schemes only for the computation of smooth solutions, non-linear flux limiters are not introduced. The centered spatial discretization is second order accurate and reads:

$$
\left\{\begin{array}{l}
\left(\frac{\mathbf{v}_{i+1 / 2}-\mathbf{v}_{i-1 / 2}}{\Delta x}\right)_{c e n t}=\frac{1}{2 \Delta x}\left(\mathbf{v}_{i+1}-\mathbf{v}_{i-1}\right) \\
\left(\frac{\psi_{i+1 / 2}-\psi_{i-1 / 2}}{\Delta x}\right)_{c e n t}=\frac{1}{2 \Delta x}\left(\psi_{i+1}-\psi_{i-1}\right) .
\end{array}\right.
$$

\subsection{Implicit time discretization}

To ensure that the centered discretization (7) is stable and to avoid the acoustic CFL, we propose a fully implicit relaxation scheme, instead of adopting classic explicit relaxation schemes $[5,8]$. The implicit scheme at first order reads:

$$
\left\{\begin{array}{l}
\frac{\psi_{i}^{n+1}-\psi_{i}^{n}}{\Delta t}+\frac{\mathbf{v}_{i+1 / 2}^{n+1}-\mathbf{v}_{i-1 / 2}^{n+1}}{\Delta x}=\mathbf{Q}\left(\psi_{i}^{n+1}\right) \\
\frac{\mathbf{v}_{i}^{n+1}-\mathbf{v}_{i}^{n}}{\Delta t}+\mathbf{A} \frac{\psi_{i+1 / 2}^{n+1}-\psi_{i-1 / 2}^{n+1}}{\Delta x}=\frac{1}{\varepsilon}\left(\mathbf{F}\left(\psi_{i}^{n+1}\right)-\mathbf{v}_{i}^{n+1}\right),
\end{array}\right.
$$

which is a simple backward Euler. For a second order implicit approximation, a BDF (Backward Differentiation Formula) second order accurate is employed.

The implicit non linear part in the source is dealt with applying one iteration of the Newton method, namely the fluxes are approximated with the Taylor expansion

$$
\mathbf{F}\left(\psi^{n+1}\right)=\mathbf{F}\left(\psi^{n}\right)+\mathbf{F}^{\prime}\left(\psi^{n}\right)\left(\psi^{n+1}-\psi^{n}\right),
$$

where $\mathbf{F}^{\prime}\left(\psi^{n}\right)$ is the Jacobian of the flux and can be computed analytically. This constitutes a significant simplification with respect to the use of complex solvers that deal with non-linear fluxes inside implicit schemes. When a non-linear source term $\mathbf{Q}(\psi)$ is present, it can be treated in the same way. With linearization (9), the derived implicit scheme possesses the following zero relaxation limit $\varepsilon \rightarrow 0^{+}$

$$
\frac{\psi_{i}^{n+1}-\psi_{i}^{n}}{\Delta t}+\frac{\mathbf{F}\left(\psi_{i+1 / 2}^{n+1}\right)-\mathbf{F}\left(\psi_{i-1 / 2}^{n+1}\right)}{\Delta x}=\mathbf{Q}\left(\psi_{i}^{n+1}\right),
$$

which is a consistent and stable discretization of (1).

In this framework, the full linear system to be solved reads 


$$
\left\{\begin{array}{l}
\mathbf{M} \Psi^{n+1}+\mathbf{N} \mathbf{V}^{n+1}=\mathbf{r} \\
\mathbf{P} \Psi^{n+1}+\mathbf{R} \mathbf{V}^{n+1}=\mathbf{s},
\end{array}\right.
$$

where $\Psi^{n+1}$ and $\mathbf{V}^{n+1}$ are the vectors containing the grid point values of the conservative and of the relaxation variables respectively. In 1D problems, $\mathbf{M}, \mathbf{N}, \mathbf{P}$ and $\mathbf{R}$ have tridiagonal sub-blocks for first order discretizations. Linearization (9) only adds terms on the diagonals of the sub-blocks of $\mathbf{P}$, not increasing the computational effort in the inversion algorithms.

The scheme is unconditionally stable. The acoustic Courant number is defined as $v_{a c}=\mu_{\max } \Delta t / \Delta x$ and with the proposed implicit scheme it can be taken significantly larger than one. This is very useful in terms of computational time when simulating low Mach flows, since the acoustic waves are extremely fast (for Euler equations $\mu_{\max } \geq|u+c|$ from condition (3), $u$ being the flow velocity and $c$ the sound speed). Therefore, the time step $\Delta t$ is not excessively limited as it would be with an explicit scheme requiring $v_{a c}<1$ for stability. Moreover, in approximating material waves, a "material CFL condition" can be employed: the material Courant number is defined as $v_{\text {mat }}=\mu_{\text {mat }} \Delta t / \Delta x$ with $\mu_{\text {mat }} \geq|u|$. To ensure accuracy on the material waves, the time step is chosen by imposing $v_{\text {mat }} \leq 1$. Thus, $\Delta t$ is not constrained by the speed of the acoustic waves but only by the speed of the material wave.

\section{Numerical results}

The proposed scheme is applied to the simulation of gas and liquid flows, governed by the one dimensional Euler equations with a generalized equation of state. In all simulations we employ $\varepsilon=10^{-8}$. Initial and boundary conditions on the relaxation variables are set to be consistent with the equilibrium state $\mathbf{v}=\mathbf{F}(\psi)$ [5].

\subsection{Steady state example: nozzle flow}

We validate the scheme by computing the steady state of a Laval nozzle flow in the quasi-1D approximation. In the general formulation (1), the conservative variables, the fluxes and the source term are:

$$
\psi=\left[\begin{array}{c}
S \rho \\
S \rho u \\
S E
\end{array}\right], \quad \mathbf{F}(\psi)=\left[\begin{array}{c}
S \rho u \\
S\left(\rho u^{2}-p\right) \\
S(E-p) u
\end{array}\right], \quad \mathbf{Q}(\psi)=\left[\begin{array}{c}
0 \\
p \partial_{x} S \\
0
\end{array}\right],
$$

where $\rho$ is the density, $u$ is the flow velocity, $E=\rho u^{2} / 2+\rho e$ is the total energy (sum of the kinetic part and the internal energy $e$ ) and $p$ is the pressure. $S=S(x)$ is the cross sectional area of the nozzle, which is a smooth function of the axial coordinate $x$. In this approximation, all flow variables depend on only the coordinate $x$.

The scheme is able to deal with different state laws. In particular, we simulate: 
- perfect gas flows, with the state law $e=p /(\rho(\gamma-1))\left(\gamma=c_{P} / c_{V}\right.$ is the heat capacity ratio and it is equal to 1.4 for biatomic gases);

- stiffened gas flows, with the state law $e=p /(\rho(\gamma-1))+p_{\infty} / \rho$, where the $p_{\infty}$ term models the intermolecular forces. This law describes flows of liquids (the parameters for water are $\gamma=4.4$ and $p_{\infty}=6.8 \cdot 10^{8} \mathrm{~Pa}$ ).

Three different configurations of the nozzle are simulated:

1. test 1 is a perfect gas flow with Mach number in the range $M \in[0.45 ; 0.7]$. At the inlet the total pressure and temperature $P_{t o t}=1 \mathrm{~Pa}$ and $T_{\text {tot }}=1 \mathrm{~K}$ are imposed, at the outlet $p_{\text {out }}=0.9 \mathrm{~Pa}$;

2. test 2 is a perfect gas flow with Mach number in the range $M \in[4 ; 9] \cdot 10^{-3}$. At the inlet $P_{t o t}=1 \mathrm{~Pa}$ and $T_{\text {tot }}=1 \mathrm{~K}$ are imposed, at the outlet $p_{\text {out }}=0.99999 \mathrm{~Pa}$;

3. test 3 is a water flow with Mach number in the range $M \in[7.26 ; 8.67] \cdot 10^{-5}$. At the inlet $P_{\text {tot }}=10 \mathrm{~Pa}$ and $T_{\text {tot }}=280 \mathrm{~K}$ are imposed, at the outlet $p_{\text {out }}=1 \mathrm{~Pa}$.

Tests 2 and 3 are low Mach flows, with the last one being almost incompressible. We remark that pressure and temperature values are here chosen with the only purpose of demonstrating the properties of the scheme.

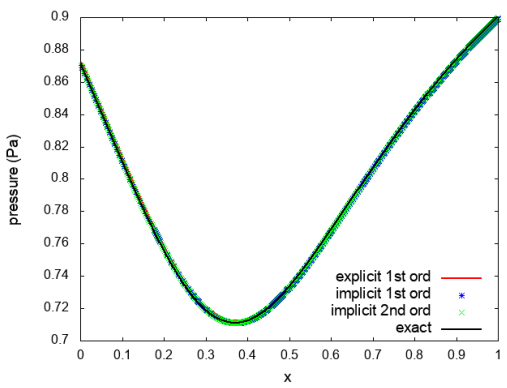

(a) Test 1

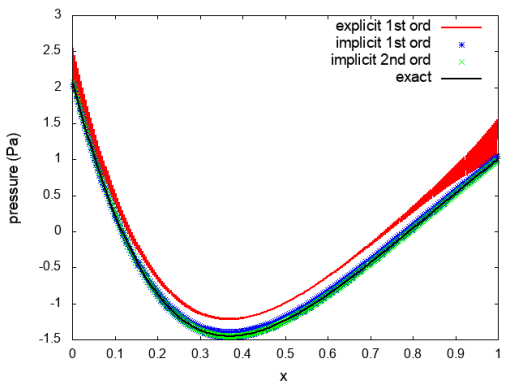

(c) Test 3

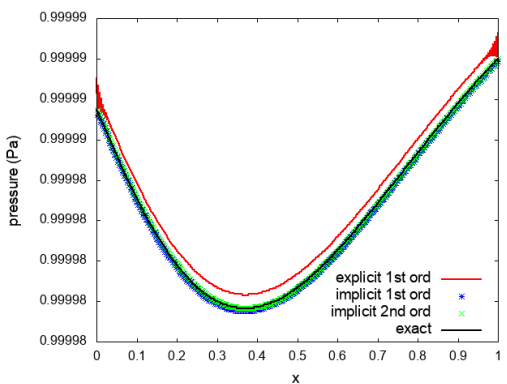

(b) Test 2

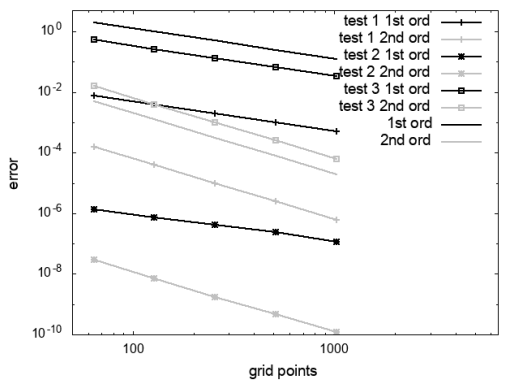

(d) Errors

Fig. 1 Nozzle flow: pressure profiles obtained with first and second order implicit schemes and with first order explicit-upwind relaxation scheme of [5] (500 grid points). Panel (d): convergence analysis of the implicit scheme for the three tests. 
The pressure profiles obtained with the first and second order implicit scheme are shown in Figs. 1(a)-1(b)-1(c) for the three tests and they are compared to the results of the classic explicit-upwind relaxation scheme of [5]. The comparison shows that for test 1 the two schemes are equivalent. Instead, for low Mach flows (tests 2 and 3 ) our implicit scheme is more accurate than the explicit one, which gives a solution shifted from the exact one and presents oscillations, due to the wrong numerical viscosity. The implicit scheme computes pressure profiles that are superimposed to the exact solution in all the three cases thanks to discretization (5). In Fig. 1(d), the convergence analysis for the implicit scheme is carried out and the correct convergence rates are recovered.

The time step for the implicit scheme is chosen by enforcing $v_{a c}=100$ at first order and $v_{a c}=40$ at second order. Thanks to this constraint, our implicit scheme is computationally less demanding. For example in test 2 , a precision of order $2 \cdot 10^{-7}$ is obtained by the first order implicit scheme in $64 \mathrm{~s}$ of CPU time and by the explicit in $3464 \mathrm{~s}$.

\subsection{Material wave simulation: gas tube}

We give a numerical illustration of the performances of the scheme for the simulation of material waves, by solving a low Mach perfect gas flow in a tube. The Euler system in the general formulation (1) reads

$$
\psi=\left[\begin{array}{c}
\rho \\
\rho u \\
E
\end{array}\right], \quad \mathbf{F}(\psi)=\left[\begin{array}{c}
\rho u \\
\rho u^{2}-p \\
(E-p) u
\end{array}\right], \quad \mathbf{Q}(\psi)=\left[\begin{array}{l}
0 \\
0 \\
0
\end{array}\right]
$$

Let $x_{d}$ be the discontinuity position, which is in the middle of the tube when $t=0$. The Riemann problem initial data are $\rho_{L}=\rho_{R}=1 \mathrm{Kg} / \mathrm{m}^{3}, u_{L}=0 \mathrm{~m} / \mathrm{s}$, $u_{R}=0.008 \mathrm{~m} / \mathrm{s}, p_{L}=0.4 \mathrm{~Pa}$ and $p_{R}=0.399 \mathrm{~Pa}$. The solution consists in two fast expansion waves and a very slow material wave. On this latter wave we have $M \simeq 6 \cdot 10^{-3}$. The results at time $t=0.25 \mathrm{~s}$ are shown in Fig. 2(a): the wave has moved from $x_{d}=0.5$ to $x_{d}=0.501167 \mathrm{~m}$, namely, for a grid spacing $\Delta x=10^{-3}$, it has crossed 1 cell. The length of this tube is $1 \mathrm{~m}$. In order to see the material wave move, we run a simulation for long times: at time $t=150$ s (Fig. 2(b)) the wave has moved from $x_{d}=200$ to $x_{d}=200.7 \mathrm{~m}$, namely, for a grid spacing $\Delta x=10^{-1}$, it has crossed 7 cells. The length of this second tube is $400 \mathrm{~m}$.

The results obtained using the implicit scheme with $v_{\text {mat }}=0.2$ (giving $\Delta t \simeq 2.2$. $10^{-2}$ on the chosen grid) are compared to the ones of the explicit-upwind relaxation scheme of [5], where instead an acoustic condition has to be enforced $\left(v_{a c}=0.4\right.$ giving $\Delta t \simeq 5 \cdot 10^{-4}$ ). Due to the material constraint on $\Delta t$, the implicit scheme is of course very diffusive on the acoustic waves. If accuracy on the rarefactions is needed, an acoustic CFL has to be enforced, thus recovering the same precision of the explicit scheme on these waves. Nevertheless, the implicit scheme is able to accurately reproduce and keep the contact wave sharp as it moves to the right, 
thanks to spatial discretization (5). The explicit scheme, instead, is very diffusive on this wave, which is completely smeared after some time (see Fig. 2(b)).

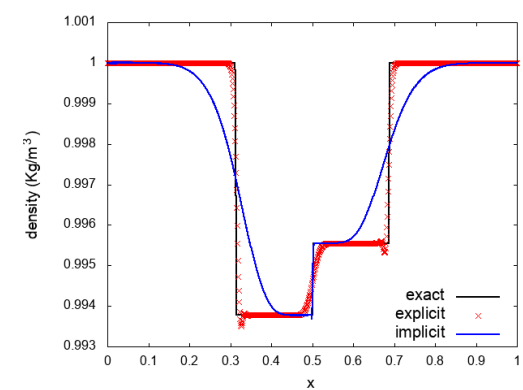

(a) Final time $t=0.25 \mathrm{~s}$

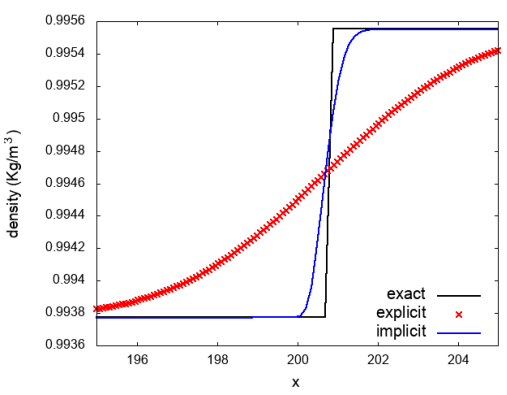

(b) Final time $t=150 \mathrm{~s}$

Fig. 2 Low Mach gas tube flow: density profiles obtained with the implicit scheme and with the explicit-upwind relaxation scheme of [5], both at first order.

\section{Conclusions}

We presented a novel implicit relaxation scheme, able to simulate low Mach flows, with first and second order accuracy. The low Mach limit is well reproduced in steady state problems and in the approximation of material waves. The scheme is simple and can be easily adapted to different state laws. In the future, simulation of waves in compressible elastic materials will be exploited.

\section{References}

1. Chalons, C., Coquel, F., Marmignon, C.: Well-balanced time implicit formulation of relaxation schemes for the Euler equations. SIAM Journal on Scientific Computing 30(1), 394-415 (2008)

2. Cordier, F., Degond, P., Kumbaro, A.: An asymptotic-preserving all-speed scheme for the Euler and Navier-Stokes equations. Journal of Computational Physics 231(17), 5685-5704 (2012)

3. Degond, P., Tang, M.: All speed scheme for the low Mach number limit of the isentropic Euler equation. arXiv preprint arXiv:0908.1929 (2009)

4. Guillard, H., Viozat, C.: On the behaviour of upwind schemes in the low Mach number limit. Computers \& fluids 28(1), 63-86 (1999)

5. Jin, S., Xin, Z.: The relaxation schemes for systems of conservation laws in arbitrary space dimensions. Communications on Pure and Applied Mathematics 48(3), 235-276 (1995)

6. Klainerman, S., Majda, A.: Compressible and incompressible fluids. Communications on Pure and Applied Mathematics 35(5), 629-651 (1982)

7. Klein, R.: Semi-implicit extension of a Godunov-type scheme based on low Mach number asymptotics i: One-dimensional flow. Journal of Computational Physics 121(2), 213-237 (1995)

8. Pareschi, L., Russo, G.: Implicit-explicit Runge-Kutta schemes and applications to hyperbolic systems with relaxation. Journal of Scientific Computing 25(1-2), 129-155 (2005) 